\title{
TWO NOTES ON MEASURE THEORY
}

\section{EDWIN HEWITT}

I. In a recent paper [1], ${ }^{1}$ Saks has indicated a construction whereby a Carathéodory outer measure can be produced on any compact metric space $M$, provided that a certain linear functional $\Phi$ is defined on the set 5 of all continuous real-valued functions whose domain is $M$. The functional $\Phi$ is required to be non-negative for non-negative functions, and to have the property that if the sequence $\left\{f_{n}\right\}$ has the uniform limit 0 , then the sequence $\Phi\left(f_{n}\right)$ is a null-sequence. (The measure itself can be defined without this last property.) The purpose of this note is to show that such a linear functional always exists, in a non-trivial form, specifically, so that $\Phi(1)=1$.

We consider the set $\mathbb{E}$ as a linear space, and together with $\mathbb{E}$ the linear space $\Re \subset \mathbb{C}$, where $\Omega$ consists of all constant functions. On the entire space $\mathbb{E}$, we define a functional $p(f)=\sup _{x \in M} f(x)$. This least upper bound always exists, since $M$, being a compact metric space, is a bicompact space, on which every continuous real-valued function is bounded. It is easy to verify that $p(f+g) \leqq p(f)+p(g)$, for all $f, g \in \mathbb{C}$, and that $p(t f)=t p(f)$ whenever $t$ is a non-negative real number. We define a linear functional $\Phi$ on the subspace $\Omega$ as follows: $\Phi(f)=f(x)$ for an arbitrary $x \in M$. It is clear that $\Phi(f)=p(f)$ for $f \in \Omega$ and that $\Phi$ is linear on $\Re$. By virtue of the celebrated theorem of Hahn-Banach, it appears that $\Phi$ can be extended linearly to all of $\mathbb{C}$ in such a fashion that $\Phi(f) \leqq p(f)$ for all $f \in \mathfrak{C}$. We further observe that $\Phi$ may be taken non-negative for non-negative functions. For, if $\Phi$ has been defined by the Hahn-Banach construction for all $f \in \mathfrak{B}$, where $\mathfrak{R} \subset \mathfrak{B} \subset \mathfrak{C}, \mathfrak{B} \neq \mathfrak{C}$, and if $g \in \mathfrak{C}-\mathfrak{B}$ and $g \geqq 0$, then the number $a=\inf _{f} \in \mathfrak{B}(p(f+g)-\Phi(f))$ is an upper bound to possible values for $\Phi(g) . a$, however, is plainly non-negative, so that $\Phi(g)$ may always be taken non-negative. Suppose now that the sequence of functions $\left\{f_{n}\right\}$ has the uniform limit 0 . The function $\epsilon-f_{n}$ is nonnegative for all $n>N(\epsilon), N(\epsilon)$ being some natural number dependent upon the arbitrary positive number $\epsilon$. Accordingly, $\Phi\left(\epsilon-f_{n}\right)=\Phi(\epsilon)$ $-\Phi\left(f_{n}\right)=\epsilon \Phi(1)-\Phi\left(f_{n}\right)=\epsilon-\Phi\left(f_{n}\right) \geqq 0$. Likewise, it is easy to show that $\epsilon+\Phi\left(f_{n}\right) \geqq 0$ for all sufficiently large $n$. It follows at once that $\lim _{n \rightarrow \infty} \Phi\left(f_{n}\right)=0$. It is proved in Saks [1] that the functional $\Phi$ can

Received by the editors January 7, 1943.

1 Numbers in brackets refer to correspondingly numbered articles in the bibliography at the end of the paper. 
be used to define a Carathéodory outer measure under which every Borel set is measurable.

II. The present note has.as its object the proof of the following result.

THEOREM. If $E$ is any infinite set, there exists a non-negative realvalued function $\Gamma$ defined on all subsets of $E$ such that:

(1) $\Gamma(A \cup B) \leqq \Gamma(A)+\Gamma(B)$;

(2) $\Gamma(A) \leqq \Gamma(B)$ if $A \subset B$;

(3) $\Gamma\left(\sum_{n=1}^{\infty} A_{n}\right)=\sum_{n=1}^{\infty} \Gamma\left(A_{n}\right)$ if $A_{n} \cap A_{m}=0$ for $m \neq n$;

(4) $\Gamma(E)=1$;

(5) $\Gamma(0)=0$;

(6) the function $\Gamma$ assumes an infinite number of different values;

(7) $\Gamma(\{p\})=0$ for all points $p \in E$ except for those in a countable subset of $E$.

Since Ulam has proved that a function enjoying properties (4) and (3) cannot vanish for all subsets containing exactly one point (where $E$ has any of a wide class of cardinal numbers), it appears that the present theorem is the strongest result possible.

The proof of this theorem depends upon a consideration of the family $\mathfrak{B}$ of all bounded real-valued functions defined on the set $E$. As in the preceding note, it is easy to prove the existence of a linear functional $\Phi$ defined on the family $\mathfrak{B}$ considered as a linear space. The construction, for our present purposes, will be considered in more detail. Let $E$ be partitioned into $\boldsymbol{\aleph}_{0}$ disjoint sets $E_{1}, E_{2}, E_{3}, \cdots$, $E_{n}, \cdots$, each having cardinal number equal to the cardinal number of $E$. Let $\omega_{n}$ be the characteristic function of the set $E_{n}$. It is obvious that $\omega_{n} \in \mathbb{B}$ for every $n$. We shall first define the linear functional $\Phi$ on the linear spaces $\mathfrak{P}_{1}, \mathfrak{P}_{2}, \mathfrak{P}_{3}, \cdots, \mathfrak{P}_{n}, \cdots$ obtained from $\Re$, the space of constant functions, by adjoining $\omega_{1}, \omega_{2}, \omega_{3}, \cdots, \omega_{n}, \cdots$ in succession and forming all possible linear combinations. As in the preceding note, we define $p(f)$ as $\sup _{x \in E} f(x)$, and $\Phi(f)$ as $f(x)$ for $f \in \Omega, x$ being any point of $E$. By the Hahn-Banach construction, if $\Phi(f)$ is to be bounded by $p(f)$, we must have, when we calculate $\Phi\left(\omega_{1}\right), a_{1} \leqq \Phi\left(\omega_{1}\right) \leqq b_{1}$, where $a_{1}=\sup _{f} \in \Re\left(-p\left(-f-\omega_{1}\right)-\Phi(f)\right)$ and $b_{1}=\inf _{f \in \Re}\left(p\left(f+\omega_{1}\right)-\Phi(f)\right)$. It is easy to show that $b_{1}=1$ and that $a_{1}=0$. We may, then, in accordance with the Hahn-Banach construction, take $\Phi\left(\omega_{1}\right)$ as $1 / 2$.

The numbers $a_{2}=\sup _{f \in \mathfrak{P}_{1}}\left(-p\left(-f-\omega_{2}\right)-\Phi(f)\right)$ and $b_{2}=\inf _{f} \in \mathfrak{P}_{1}$ $\left(p\left(f+\omega_{2}\right)-\Phi(f)\right)$ are lower and upper bounds, respectively, for $\Phi\left(\omega_{2}\right), a_{2}$ may be computed as 0 , and $b_{2}$, as it is easy to see, is equal 
to $1 / 2$. We may thus put $\Phi\left(\omega_{2}\right)=1 / 4$. This process may be continued by finite induction; it is found that the function $\omega_{n}$ may be assigned the value $1 / 2^{n}$ under the functional $\Phi$. $\Phi$ having been defined for the linear space generated by $\Re$ and the sequence $\left\{\omega_{n}\right\}$, the HahnBanach construction is carried out for the rest of $\mathfrak{B}$ in any fashion consonant with the restrictions of that theorem, provided that $\Phi(f) \geqq 0$ for non-negative functions $f$. We thus have a linear nonnegative functional defined on all of the space $\mathfrak{B}$.

The measure $\Gamma(A)$ for every subset $A$ of $E$ can now be defined: $\Gamma(A)=\Phi\left(\omega_{A}\right), \omega_{A}$ being the characteristic function of the set $A$. Properties (1)-(7) can now be established. It is plain that $\omega_{A \cup B} \leqq \omega_{A}+\omega_{B}$, whence $\Phi\left(\omega_{A}+\omega_{B}-\omega_{A \cup B}\right) \geqq 0$, and consequently $\Phi\left(\omega_{A \cup B}\right) \leqq \Phi\left(\omega_{A}\right)+\Phi\left(\omega_{B}\right)$, which inequality establishes (1). It is also obvious that $A \subset B$ implies that $\omega_{A} \leqq \omega_{B}$. From this, we infer property (2).

We examine (3) in some detail. If $A$ and $B$ are disjoint sets, it follows that $\omega_{A \cup B}=\omega_{A}+\omega_{B}$, and consequently $\Phi\left(\omega_{A \cup B}\right)=\Phi\left(\omega_{A}\right)$ $+\Phi\left(\omega_{B}\right)$, that is, the measure is additive for all subsets of $E$. We may thus state that all subsets of $E$ are measurable in the sense of Carathéodory. It is easy to prove from this fact that if $\left\{A_{n}\right\}$ is any sequence of pairwise disjoint sets, then $\Gamma\left(\sum_{n=1}^{\infty} A_{n}\right)=\sum_{n=1}^{\infty} \Gamma\left(A_{n}\right)$. The proof may be carried over word for word from a similar proof in Saks [2, chap. 2, $\$ 4$, p. 44, Theorem 4.1].

Statements (4), (5), and (6) are immediate consequences of the definitions of $\Phi$ and $\Gamma$.

To prove that $\Gamma$ vanishes for all points except those in a countable subset, we assume the contrary. If an uncountable set $T$ of points exist such that $\Gamma(\{p\})>0$ for every $p \in T$, then there is some $\epsilon>0$ with $\Gamma\left(\left\{p_{n}\right\}\right)>\epsilon$, where $p_{n} \in T$, and $n=1,2,3, \cdots$. On account of property (3), we have $\Gamma\left(\sum_{n=1}^{\infty}\left\{p_{n}\right\}\right)=\sum_{n=1}^{\infty} \Gamma\left(\left\{p_{n}\right\}\right)=\infty$. Since $1=\Gamma(E) \geqq \Gamma\left(\sum_{n=1}^{\infty}\left\{p_{n}\right\}\right)$, a contradiction is apparent.

\section{BiBLIOGRAPHY}

1. S. Saks, Integration in abstract spaces, Duke Math. J. vol. 4 (1938) pp. 408-411.

2. - Theory of the integral, Monografie Matematyczne, Warszawa, 1937.

3. S. Ulam, Zur Masstheorie in der allgemeinen Mengenlehre, Fund. Math. vol. 16 pp. 140-150.

HARVARD UNIVERSITY 